\title{
Theoretical Calculation and Research on the Strength of a Artillery Barrel
}

\author{
Jianlin Zhong,Wenjia Xia and Jie Ren \\ School of Mechanical Engineering,Nanjing University of Science and Technology,Nanjing \\ 210094,Jiangsu,China \\ zhongjianlin@njust.edu.cn,15705420443@163.com,renjie@njust.edu.cn
}

Keywords : Theoretical Calculation ,Artillery Barrel, Strength

Abstract.The theoretical calculation method of gun barrel strength is put forward.First of all,get the stress function solutions of the plane axisymmetric problems based on equilibrium, geometry and physical equations. Secondly, the radial theoretical formula for the stress, strain and displacement of the barrel of artillery is deduced.Finally,get an elastic design scheme for the barrel and establish the simulation verification of the finite element model of the design scheme. The results show that the finite element calculation and the theoretical calculation are in good agreement, which verifies the correctness of the theoretical derivation.

\section{Introduction}

The barrel is a tube that gives the projectile a muzzle velocity and a shot when the gun launches.The barrel is usually simplified to a thick-walled cylinder for research.This paper wil simplify the bore pressure of the barrel into an axisymmetric problem of plane stress and introduce the stress function based on equilibrium, geometry and physical equations to research it.Deduce the radial theoretical formula for the stress, strain and displacement of the barrel of artillery.Giving an elastic design scheme for the barrel,establishing the finite element model of the design scheme and verifying the correctness of theoretical calculation.

\section{The Stress Function Solutions of Plane Axisymmetric Problems}

The Basic equations of plane axisymmetric problems. The axial stress of artillery barrel is small so that its effect on the strength is minimal, making it as the plane stress and axisymmetric problems. There is only radial displacement $u$ in polar coordinate system,and $u$ is only a function of the radius. The circumferential displacement $v=0$, so there are only radial strain and circumferential strain, and the tangential straing $\gamma_{r} \theta=0$. As shown in Figure 1 , select a circumferential linear $P B$ whose radius is $r$ and a radial linear element $P A$ whose length is $d r$, the two line element will move to $P B$ and $P A$ after the deformation.

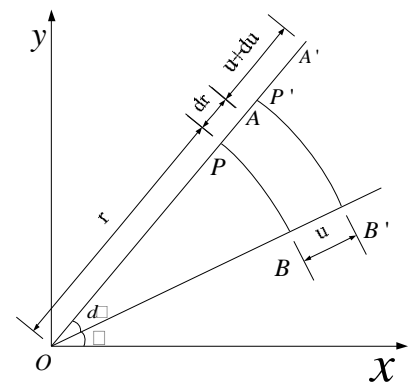

Fig.1 Displacement and strain

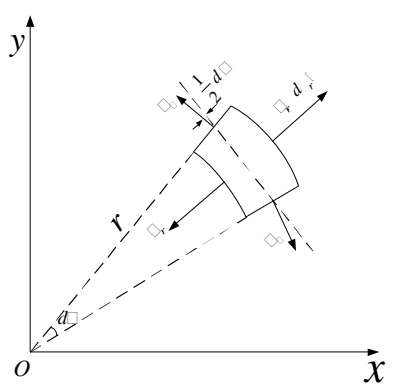

Fig.2 equilibrium equation

Get strain as:

$$
x_{r}=\frac{P^{\prime} A^{\prime}-P A}{P A}=\frac{(\mathrm{u}+\mathrm{du})-\mathrm{u}}{d r}=\frac{d u}{d r},
$$




$$
x_{q}=\frac{P^{\prime} B^{\prime}-P B}{P B}=\frac{(\mathrm{r}+\mathrm{u}) \mathrm{d} q-r d q}{r d q}=\frac{u}{r} .
$$

The geometric equation is:

$$
x_{r}=\frac{d u}{d r}, x_{q}=\frac{u}{r} \text {. }
$$

For axisymmetric problem of plane stress,radial stress $\sigma_{r}$ and circumferential stress $\sigma_{\theta}$ are functions of radius $r$.As shown in Figure 2,in an element that is cut out of a radial plane and a circumferential surface, the circumferential equilibrium fulfils automatically, the radial equilibrium relationship is:

$$
\left(s_{r}+\mathrm{d} s_{r}\right)(\mathrm{r}+\mathrm{dr}) \mathrm{d} q-s_{r} r d q-2 s_{q} d r \sin \left(\frac{1}{2} d q\right)=0 .
$$

Get the equilibrium equation after simplifying:

$$
\frac{d s_{r}}{d r}+\frac{s_{r}-s_{q}}{r}=0 \text {. }
$$

For axisymmetric problem of plane stress, its physical equation is:

$$
\begin{aligned}
& x_{r}=\frac{1}{E}\left(s_{r}-m s_{q}\right) \\
& x_{q}=\frac{1}{E}\left(s_{q}-n s_{r}\right)
\end{aligned}
$$

Plane stress axisymmetric stress function solution. When solving the problem of elastic mechanics, it is necessary to solve geometric equation, equilibrium equation and physical equation at the same time, and the solution must fulfil the boundary condition.

Equation (5) can be further written as:

$$
\frac{d}{d r}\left(r s_{r}\right)=s_{q} \text {. }
$$

Introduction of stress function $\varphi(r)$.Combination of:

$$
r s_{r}=j(\mathrm{r}), \frac{d j}{d r}=s_{q} .
$$

And equation (3),(6)and(8) will lead to:

$$
r \frac{d^{2} j}{d r^{2}}+\frac{d j}{d r}-\frac{j}{r}=0 \text {. }
$$

Equation (9) can be converted into the following integral form:

$$
r \frac{d}{d r}\left(\frac{1}{r} \frac{d}{d r}(r j)\right)=0 \text {. }
$$

The following equation will be gotten by integral the above equation:

$$
j=A r+\frac{B}{r}
$$

Among them, $A$ and $B$ are integral constant.Plug $\varphi$ into the equation (8), the relation between stress, strain, displacement and radius can be gotten by combining equation (3) and (6):

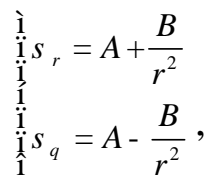

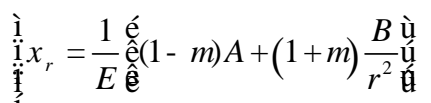

$$
\begin{aligned}
& \stackrel{1}{\dddot{i}} x_{q}=\frac{1}{E} \frac{\hat{\mathrm{e}}}{\hat{\mathrm{e}}}(1-m) A-(1+m) \frac{B}{r^{2}} \stackrel{\mathrm{u}}{\mathrm{u}}
\end{aligned}
$$

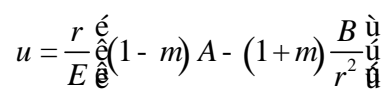

At this point, the stress, strain and displacement of the plane stress axisymmetric problem are obtained, and the integral constant can be obtained according to the boundary condition. 


\section{Analysis and calculation of the strength of barrel}

The barrel can be reducible to a thick-walled cylinder when researching it.As shown in Figure 3 ,the inner radius and outer radius of the thick-walled cylinder is $a$ and $b$. The internal pressure on the inner surface is $p_{1}$ and the external pressure on the outer surface is $p_{2}$. The corresponding boundary conditions are $\left.\sigma_{r}\right|_{r=a}=-p_{1}$ and $\left.\sigma_{r}\right|_{r=b}=-p_{2}$, plug the above two boundary conditions into equation12,two constants will be gotten:

$$
A=\frac{a^{2} p_{1}-b^{2} p_{2}}{b^{2}-a^{2}}, B=\frac{a^{2} b^{2}\left(p_{2}-p_{1}\right)}{b^{2}-a^{2}} \text {. }
$$
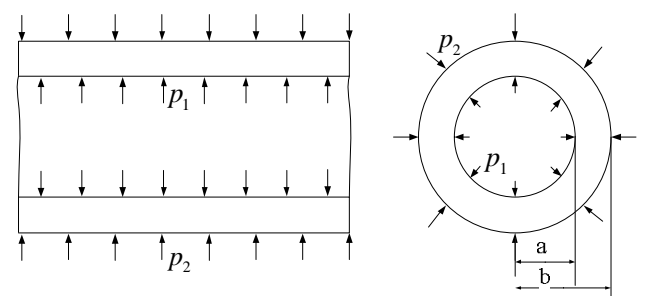

Fig.3 Pressure equalizing thick cylinder

Plug equation15 into equation (12) to (14) can get the stress component (Lame formula), strain components and displacement components:

$$
\begin{aligned}
& \begin{array}{l}
\grave{i} s_{r}=\frac{a^{2} p_{1}-b^{2} p_{2}}{b^{2}-a^{2}}+\frac{a^{2} b^{2}\left(p_{2}-p_{1}\right)}{r^{2}\left(b^{2}-a^{2}\right)} \\
\vdots \\
i s_{q}=\frac{a^{2} p_{1}-b^{2} p_{2}}{b^{2}-a^{2}}-\frac{a^{2} b^{2}\left(p_{2}-p_{1}\right)}{r^{2}\left(b^{2}-a^{2}\right)}, \\
i_{1}
\end{array} \\
& \text { ì } x_{r}=\frac{1}{E} \underset{\hat{\hat{e ̂}}}{\hat{\hat{e}}(1-m)} \frac{a^{2} p_{1}-b^{2} p_{2}}{b^{2}-a^{2}}+(1+m) \frac{a^{2} b^{2}\left(p_{2}-p_{1}\right)}{r^{2}\left(b^{2}-a^{2}\right)}
\end{aligned}
$$

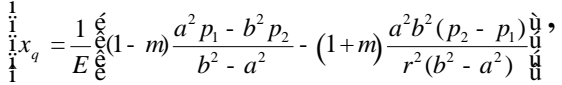

$$
\begin{aligned}
& u=\frac{r}{E} \text { ề }
\end{aligned}
$$

Using the second strength theory based on elastic failure theory of material to do elastic design of barrel,the failure criterion is Tresca yield criterion that the barrel reaches the limit of elastic strength when $\sigma_{r}-\sigma_{\theta}=\sigma_{s}$.

The barrel uniform only sustains internal pressure $p_{1}$, the external pressure $p_{2}$ is 0 , the stress component is:

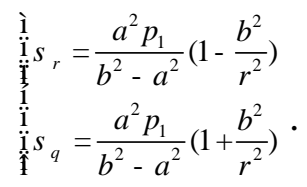

On the inner wall of a thick-walled tube, $\left(\sigma_{\theta^{-}} \sigma_{r}\right)$ has the maximum value, and it will come into the plastic state firstly.Plug $(r=a)$ into equation (19) and Tresca yield criterion:

$$
p_{e}=\frac{s_{s}}{2}\left(1-\frac{a^{2}}{b^{2}}\right) \text {. }
$$

Givin the inner diameter of barrel $a$,yield strength $\sigma_{s}$, the limit chamber pressure $p_{\varepsilon}$, we will get the outer diameter of barrel $b$ and complete the elastic strength design for barrelby from equation (20).

\section{Flexible design of barrel}

Using the average pressure curve to design the barrel. We can get the maximum bore pressure 
$p_{m}, p$ - $l$ chamber pressure curve, projectile stroke $l_{\varepsilon}$, chamber length $l_{y s}$ and so on by internal ballistic calculation. We can get the internal radius $r_{a}$ of each section by bore design.Combine main tactical technical elements such as caliber $d$ with calculation results of interior ballistics,draw the theoretical strength curve of barrel,give schematic diagram of preliminary design scheme for barrel as Figure 4 shows.

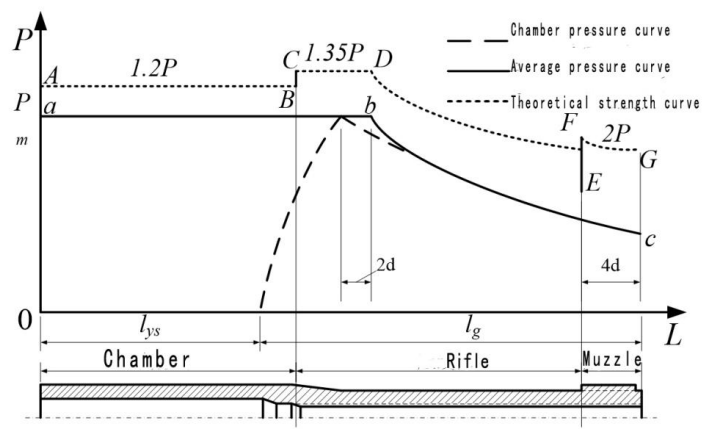

Fig.4 Schematic diagram of preliminary design scheme for barrel

In order to simplified calculation, suppose the outer diameters of the chamber in Figure 4 are the same.Give the parameters of each part of the barrel as shown inTable 1.

Table.1 Parameters of each part of barrel

\begin{tabular}{ccccccc}
\hline $\begin{array}{c}\text { The distance from the } \\
\text { bottom of the } \\
\text { chamber[mm }]\end{array}$ & $\begin{array}{c}\text { Chamber } \\
650\end{array}$ & \multicolumn{2}{c}{ Rifle } & \multicolumn{3}{c}{ Muzzle } \\
\hline$d_{1}[\mathrm{~mm}]$ & 102.3 & 66.7 & 66.7 & 66.7 & 66.7 & 66.7 \\
$p_{1}[\mathrm{Mpa}]$ & 241.6 & 271.8 & 191.2 & 201.3 & 195.7 & 192.3 \\
$d_{2}[\mathrm{~mm}]$ & 201.2 & 162.1 & 103.5 & 107.6 & 102.5 & 101.5 \\
\hline
\end{tabular}

\section{Finite element model of barrel}

Establish the finite element model of barrel design scheme,get the chamber section of the radial stress distribution as shown in Figure 5.Compared with the theoretical calculation results in equation 16, the finite element calculation is in good agreement with the theoretical calculation, proving the validity of the theoretical results.

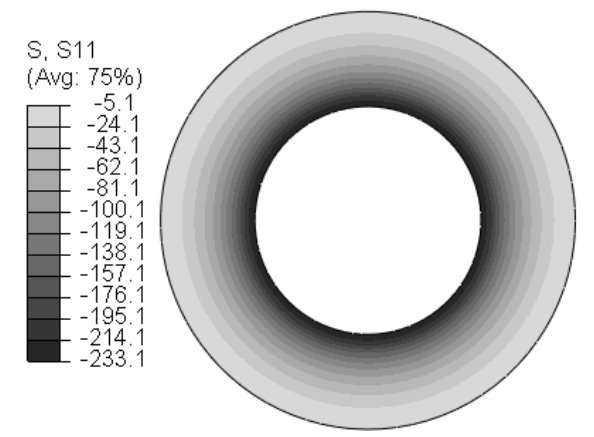

Fig.5 Radial stress distribution cloud chart 


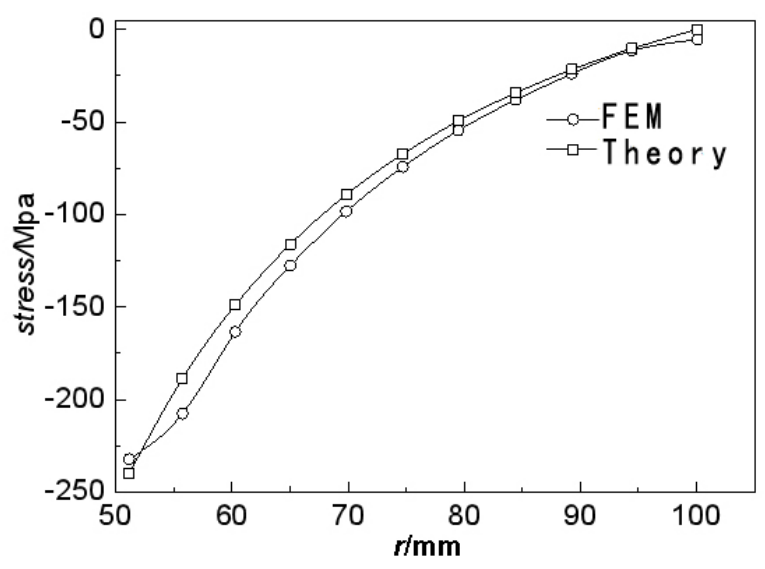

Fig.6 Comparison between finite element and theoretical calculation

\section{Conclusions}

The results of the finite element calculation and the theoretical calculation of the radial stress of the body tube are in good agreement,it shows that the derivation of the theoretical formula for the stress, strain and displacement of the body is correct

\section{Acknowledgements}

Gratefully thanks for the financial supports from Innovation Partnership Fund for Universities of China Aerospace Science and Technology Corporation (CASC) and Natural Science Funds for Young Scholar of Jiangsu Province (No. BK 201708237), my thanks also go to the experts and th e editors for their work.

\section{References}

[1] Pan Yu Tian,Guo Bao Quan,et al.Barrel Design[M] Beijing:Weaponry Industry Press,2007

[2] Li Bao Feng,Liu Xie Yi,Ni Xin Hua,et al.Application of the Twin Shear Strength Theory in Strength-Calculation of Gun Barrels[J] Science Technology and Engineering,2007, 7(13): 3235-3237

[3] Theocaris P S, A general yield criterion for engineering materials[J]. Depending on Void Growth Mechanical, 1986(21): 91-105

[4] Wu Ying.The Assembly Pressure of Circular Tubes with Different Lengths[J] Journal of Taiyuan University of Technology,2000, 31(2): 215-217

[5] Wang Ying Ze,Zhang Xiao Bing, Yuan Ya Xiong,et al.Flexible Dynamics Model Analysis of Gun Tube[J] Journal of Gun Launch \& Control,2008, (4): 49-52

[7] Di Change Chun,Wang Xin Gui,Qin Jun Qi,et al.Strength Design of Gun Barrel Based on Consistent Reliability[J] Journal of Gun Launch \& Control,2002, (2): 58-60 